\title{
Observed hierarchy of student proficiency with period, frequency, and angular frequency
}

\author{
Nicholas T. Young and Andrew F. Heckler \\ Department of Physics, Ohio State University, Columbus, Ohio 43210, USA
}

(Received 28 August 2017; published 19 January 2018)

\begin{abstract}
In the context of a generic harmonic oscillator, we investigated students' accuracy in determining the period, frequency, and angular frequency from mathematical and graphical representations. In a series of studies including interviews, free response tests, and multiple choice tests developed in an iterative process, we assessed students in both algebra-based and calculus-based, traditionally instructed university-level introductory physics courses. Using the results, we categorized nine skills necessary for proficiency in determining period, frequency, and angular frequency. Overall results reveal that, postinstruction, proficiency is quite low: only about $20 \%-40 \%$ of students mastered most of the nine skills. Next, we used a semiquantitative, intuitive method to investigate the hierarchical structure of the nine skills. We also employed the more formal item tree analysis method to verify this structure and found that the skills form a multilevel, nonlinear hierarchy, with mastery of some skills being prerequisite for mastery in other skills. Finally, we implemented a targeted, 30-min group-work activity to improve proficiency in these skills and found a 1 standard deviation gain in accuracy. Overall, the results suggest that many students currently lack these essential skills, targeted practice may lead to required mastery, and that the observed hierarchical structure in the skills suggests that instruction should especially attend to the skills lower in the hierarchy.
\end{abstract}

DOI: 10.1103/PhysRevPhysEducRes.14.010104

\section{INTRODUCTION}

Period, frequency, and angular frequency are three basic and commonly used quantities in physics, appearing in many contexts such as oscillations, waves, rotations, ac circuits, motors, and generators. A perusal of introductory physics textbooks (e.g., Ref. [1-3]) reveals that these three quantities are represented either explicitly or implicitly in both equation and graphic representations and are as fundamental to the topics of oscillations and rotations as, say, the quantity velocity is to the topic of kinematics. Yet, while student understanding of velocity in kinematics has been studied for decades (e.g., Refs. [4,5]), overall, there remain large gaps in documentation and analysis of student proficiency and difficulties with making calculations and using graphs and equations to determine period, frequency, and angular frequency. In fact, we know of only of two studies directly investigating aspects of student understanding of period, frequency, and angular frequency. Specifically, Wan et al. [6] have found that students confuse period and angular frequency when interpreting periodic functions, including functions for waves, and Turner, Ellis, and Beichner [7] found that students perform much better

Published by the American Physical Society under the terms of the Creative Commons Attribution 4.0 International license. Further distribution of this work must maintain attribution to the author(s) and the published article's title, journal citation, and DOI. on a validated test for understanding linear kinematics graphs than on an equivalent test on rotational kinematics graphs. A few less relevant studies have investigated student understanding of concepts that implicitly include some of these three quantities, such as in the study of simple harmonic motion and oscillations [8,9], wave phenomena [10-12], and rotations [13].

Therefore, this study has three main goals. The first goal is to determine student proficiency in and difficulties with using graphs and equations to determine period, frequency, and angular frequency in generic simple harmonic oscillator tasks. Achievement of the first goal provides the context for the second goal: Because the period, frequency, and angular frequency of a system are so closely related, one might reasonably expect that student proficiencies or difficulties in determining each quantity are also closely related. Therefore, our second goal is to investigate whether there are any hierarchies in student proficiencies in determining these quantities. That is, can we establish that being proficient in determining one specific quantity is necessary for being proficient in determining another? We will use both formal and more intuitive methods to demonstrate a hierarchy.

The third goal is to determine the extent to which a short group-work activity can significantly improve student proficiencies with these skills. The determination of period, frequency, and angular frequency is such a fundamental, critical set of skills for several areas of science that we consider them "essential skills" in the sense discussed by 
Mikula and Heckler [14]. That is, these skills are relatively simple procedural skills, these skills are necessary for completing more complex problems commonly found in the curriculum, and these skills are largely automated in experts. Given the relatively simple nature of these procedural skills, it is possible that only brief, targeted instruction is needed for significant improvements in proficiency (e.g., see Refs. [14,15]).

\section{STUDENT PROFICIENCIES AND DIFFICULTIES WITH PERIOD, FREQUENCY, AND ANGULAR FREQUENCY}

The goal of the first portion of the study is to determine student proficiencies and characterize difficulties with determining the period, frequency, and angular frequency from various mathematical and graphical representations.

We began this investigation by conducting small pilot studies with introductory calculus-based physics students. The students were asked to identify the period, frequency, and angular frequency from various representations and to match graphical representations with their corresponding equational representations. These pilot studies were performed using free response paper tests and student interviews. Multiple choice items and distractors used in the main study were derived from the student responses in the pilot studies.

Using the results of the pilot studies and with the essential skills practice framework in mind, we also categorized the skills for determining the period, frequency, and angular frequency from graphical and mathematical representations into nine skills, shown in Fig. 1. Two of these skills, determining the period from a graphical representation and determining the angular frequency from a mathematical representation, $x(t)=\cos (\omega t)$, can be inferred (read off) directly from the representation with no need for calculation. The next four skills require two steps: first reading off the period from a graph or the angular frequency $\omega$ from an equation, and second using the standard relations $(T=1 / f, \omega=2 \pi / T, \omega=2 \pi f)$ to calculate a different quantity (such as frequency). The final three skills require using one of the standard relations $(T=1 / f, \omega=2 \pi / T, \omega=2 \pi f)$ to determine a quantity (such as frequency) when another is given (such as period).

\section{A. Participants}

The participants in this portion of the study were students enrolled in either the first semester, introductory algebrabased physics course, the first semester introductory calculus-based physics course, or the second semester introductory calculus-based physics course at Ohio State University, a large public research university. The algebrabased course is primarily taken by life science and pre-med students while the calculus-based course is primarily taken by physical science and engineering students. These were traditionally instructed, large enrollment courses, consisting of 2-3 lectures per week, 1 recitation per week, and 1 laboratory session per week.

Our data were collected via two methods. Data from the students in the calculus-based course were collected from in-person sessions with students in our physics education research laboratory. Students signed up for a $1 \mathrm{~h}$ session during the course of the semester and received participation credit worth roughly one homework assignment. During the session, the students completed a variety of physics education tasks, including the ones described in this study. Tasks not part of this study were focused on different physics topics. Overall, approximately $90 \%$ of the students agreed to participate in our studies but only those who participated after relevant instruction on period, frequency, and angular frequency were included in this study. During the $1 \mathrm{~h}$ session, students answered physics questions on paper in a proctored test room at their own pace. Students were asked to give an honest effort and through our observations, it appears that they did.

Data from the algebra-based course were collected via an online, multiple-choice test administered through the online learning management system for the course. The test was assigned the last week of the semester before final exams and students were given one week to complete the test. Students who completed the test received credit worth approximately one homework assignment. Before starting the test, students were asked if their data could be used for research purposes. If a student declined, they were still able to complete the test for course credit. Approximately $80 \%$ of the students agreed to participate in the study.

\section{B. Materials}

Students in the calculus-based physics course were administered an assessment during their in-person session. Example items are shown in Fig. 1. The assessment consisted of 36 items, assessed students on all nine skills, and was administered after the unit on harmonic oscillators. Questions designed to test the calculation skills were presented as free response questions while the questions featuring graphical representations were only presented in multiple-choice format. The questions featuring equational representations were presented in both free response and multiple-choice format. For example, for the free response questions, students were presented with an equation of the form $x(t)=\cos (\omega t)$ (where $\omega$ was replaced with a numerical value) and asked to determine either the period, frequency, or angular frequency. In the pilot studies, we studied small variations in the "direction" the question was posed: for example, we provided the equation $x(t)=$ $\cos (\omega t)$ and asked for, say the period, or the reverse, we provide the period and asked for the correct equation $x(t)=\cos (\omega t)$ (where all cases $\omega$ was replaced with a numerical value). We found no significant differences in response pattern between these two forms, so for the 


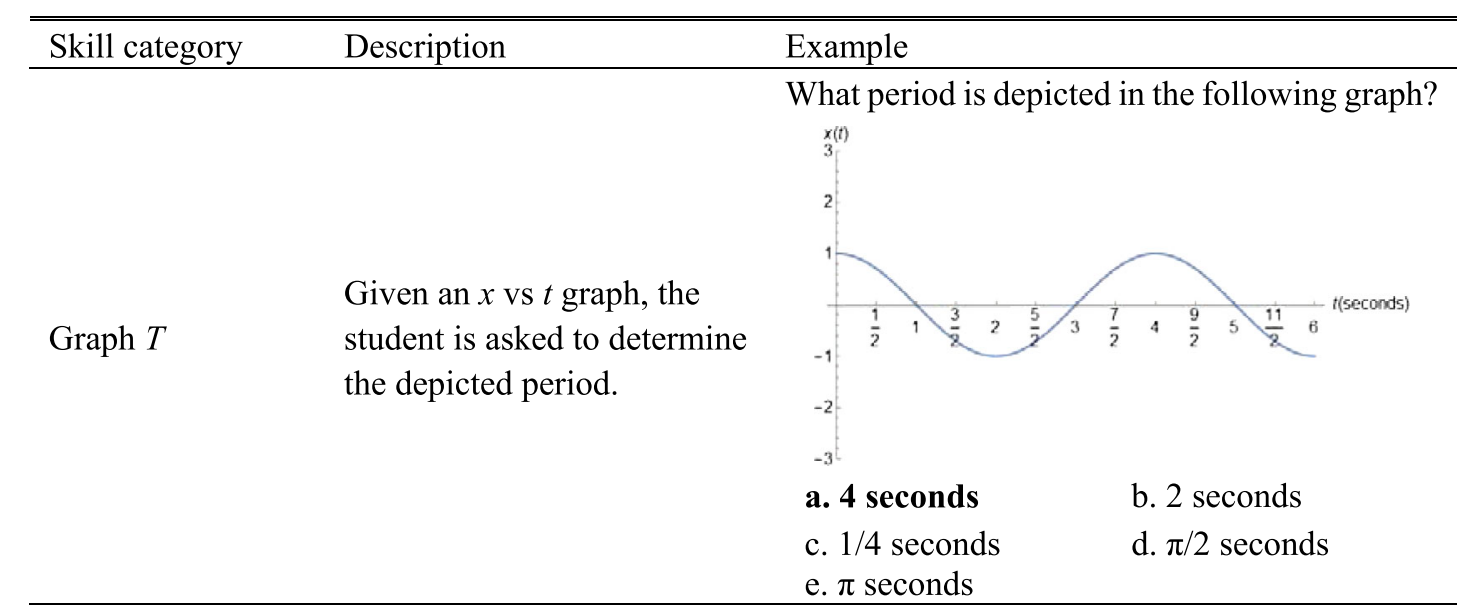

What frequency is depicted in the following graph?

Given an $x$ vs $t$ graph, the Graph $f$ student is asked to determine the depicted frequency.

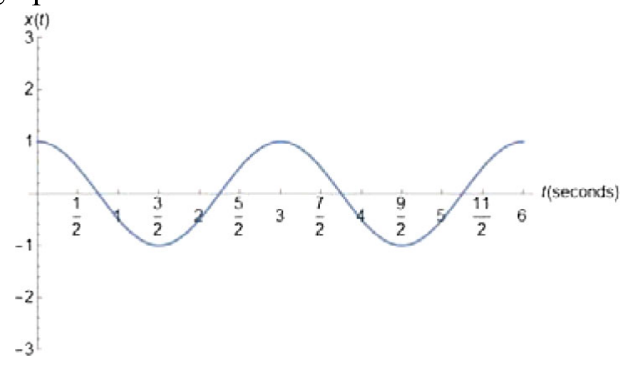
a. $3 \mathrm{~Hz}$
b. $3 / 2 \mathrm{~Hz}$
c. $1 / 3 \mathrm{~Hz}$
d. $2 \pi / 3 \mathrm{~Hz}$
e. $\pi / 3 \mathrm{~Hz}$

What angular frequency is depicted in the following graph?

Given an $x$ vs $t$ graph, the Graph $\omega$ student is asked to determine the depicted angular frequency.

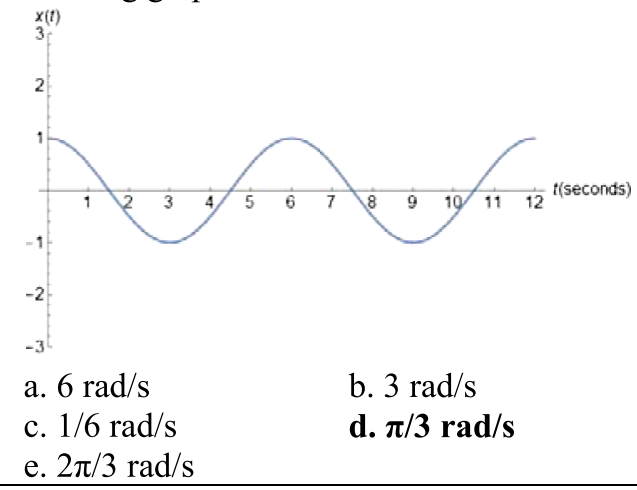

The equation $x(t)=5 \cos \left(\frac{2 \pi}{7} t+2\right)$ describes Equation $T$

Given an $x(t)$ equation, the student is asked to determine the period. the motion of a mass on a spring. What is the period of this motion, assuming $t$ is in seconds?
a. $5 \mathrm{~s}$
b. $2 \pi / 7 \mathrm{~s}$
c. $7 /(2 \pi) \mathrm{s}$
d. $7 \mathrm{~s}$

FIG. 1. The nine skill categories needed to determine the period, frequency, and angular frequency from graphical and mathematical representations.

assessments used in this paper, we included items of both types.

Students in the algebra-based physics course were administered an online, 30-item test, covering all nine skills. The test was administered after the unit on harmonic oscillators. Because of limitations in the learning management system and the number of students in the course, the entire test was made multiple choice. Since the number of 


\begin{tabular}{|c|c|c|}
\hline Equation $f$ & $\begin{array}{l}\text { Given an } x(t) \text { equation, the } \\
\text { student is asked to determine } \\
\text { the frequency. }\end{array}$ & $\begin{array}{l}\text { The equation } x(t)=7 \cos \left(\frac{2 \pi}{5} t+3\right) \text { describes } \\
\text { the motion of a mass on a spring. What is the } \\
\text { frequency of this motion, assuming } t \text { is in } \\
\text { seconds? } \\
\begin{array}{ll}\text { a. } 7 \mathrm{~Hz} & \text { b. } 2 \pi / 5 \mathrm{~Hz} \\
\text { c. } 5 /(2 \pi) \mathrm{Hz} & \text { d. } 5 \mathrm{~Hz} \\
\text { e. } 1 / 5 \mathrm{~Hz} & \end{array}\end{array}$ \\
\hline Equation $\omega$ & $\begin{array}{l}\text { Given an } x(t) \text { equation, the } \\
\text { student is asked to determine } \\
\text { the angular frequency. }\end{array}$ & $\begin{array}{l}\text { The equation } x(t)=13 \cos (14 t+11) \\
\text { describes the motion of a mass on a spring. } \\
\text { What is the angular frequency of this motion, } \\
\text { assuming } t \text { is in seconds? } \\
\begin{array}{ll}\text { a. } 13 \mathrm{rad} / \mathrm{s} & \text { b. } 14 \mathrm{rad} / \mathrm{s} \\
\text { c. } 1 / 14 \mathrm{rad} / \mathrm{s} & \text { d. } \pi / 7 \mathrm{rad} / \mathrm{s} \\
\text { e. } 7 / \pi \mathrm{rad} / \mathrm{s} & \end{array}\end{array}$ \\
\hline Calculate $T$ or $f$ & $\begin{array}{l}\text { Given the period or } \\
\text { frequency, the student is } \\
\text { asked to calculate the other } \\
\text { using } T=1 / f \text {. }\end{array}$ & $\begin{array}{l}\text { A harmonic oscillator has a period of } 5 \text { seconds. } \\
\text { What is the frequency? } \\
\text { Frequency: } \mathbf{1} / \mathbf{5 ~} \mathbf{~ H z}\end{array}$ \\
\hline Calculate $\omega$ or $T$ & $\begin{array}{l}\text { Given the period or angular } \\
\text { frequency, the student is } \\
\text { asked to calculate the other } \\
\text { using } \omega=2 \pi / T \text {. }\end{array}$ & $\begin{array}{l}\text { A harmonic oscillator has an angular frequency } \\
\text { of } 2 \pi / 3 \mathrm{rad} / \mathrm{s} \text {. What is the period? } \\
\text { Period: } 3 \text { seconds }\end{array}$ \\
\hline Calculate $\omega$ or $f$ & $\begin{array}{l}\text { Given the angular frequency } \\
\text { or frequency, the student is } \\
\text { asked to calculate the other } \\
\text { using } \omega=2 \pi f \text {. }\end{array}$ & $\begin{array}{l}\text { A harmonic oscillator has an angular frequency } \\
\text { of } \pi / 2 \mathrm{rad} / \mathrm{s} \text {. What is the frequency? } \\
\text { Frequency: } \mathbf{1} / \mathbf{4 ~ H z}\end{array}$ \\
\hline
\end{tabular}

FIG. 1. (Continued)

questions on the online test needed to be limited, the calculate questions were combined into a single item. For example, if presented with a question asking for the corresponding frequency and angular frequency for a period of $T=3 \mathrm{~s}$, the student would have to select the response with both $f=1 / 3 \mathrm{~Hz}$ and $\omega=2 \pi / 3 \mathrm{rad} / \mathrm{s}$.

For all of these tests, students who did not answer at least half of the items or who had participated in this study from a previous semester were excluded from the data analysis. Since at least half of each of the assessments was multiple-choice questions and none of the students in the study had their session end before turning in their assessment, we believe that requiring at least of the half questions to be answered serves as a proxy for the students putting in an honest effort, and their data are thus representative of a typical student. Through this process, less than $10 \%$ of the students were removed.

\section{Overall results}

The proportions of correct responses by skill are shown in Fig. 2. There are a few important general observations about the data in Fig. 2. First and most importantly, students from both populations were far from mastery on these fundamental skills: the average percentage correct for most skills ranged from about $30 \%$ to $60 \%$, even after instruction. Second, except for a few exceptions, the performance for both populations was similar. Since the assessments for the two populations were not identical, we did not conduct statistical tests for quantitative comparisons between the two populations, but inspection of Fig. 2 reveals the similarity of performance. Third, we found no significant difference in performance on the "direction" of the questions for the skills in which students must use one of the standard relations $(T=1 / f, \omega=2 \pi / T, \omega=2 \pi f)$. That is, there was no difference in performance between a question in which frequency is given and the student is asked to calculate period and the reverse question, in which period is given and the student is asked to calculate frequency. Therefore, we combined the scores for a given standard relation (e.g., $T=1 / f$ ) into a single score.

Finally, examination of Fig. 2 reveals some significant variation in performance between the skills. Overall, students performed the best on finding the period from a graphical representation and on applying the relationship $T=1 / f$. Both populations tended to perform poorly on finding the period or frequency from an equation of the 


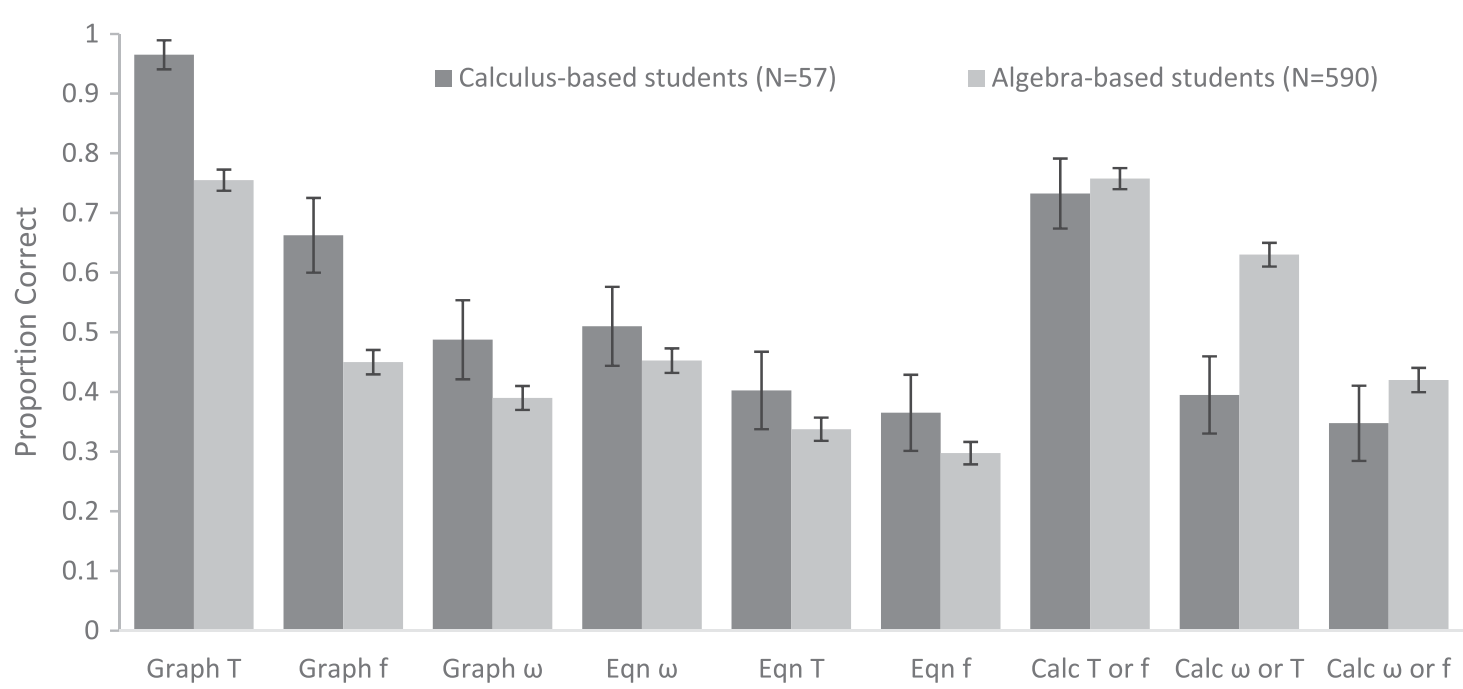

FIG. 2. The proportion of correct responses plotted for each of the nine skill categories by each population of students. Not all students saw questions from each of the nine skills.

form $x(t)=\cos (\omega t)$ and most skills involving angular frequency. The differences in performance of these skills suggest possible hierarchies in these skills, which will be discussed in Sec. III.

\section{Observed specific difficulties}

In this section we discuss the most common difficulties observed in student responses to the assessments and pilot study testing and interviews. To be included in our analysis, the difficulty had to be observed on both free response and multiple-choice questions and had to be made by at least
$10 \%$ of one of the populations. A summary of the difficulties, mean percent of students having each difficulty, the percent of students who repeatedly (at least $75 \%$ of the time) encountered the difficulty, and the percent of students who never gave an answer consistent with the difficulty is shown in Table I. The skill of relating the period and frequency using $T=1 / f$ is excluded from the table since there were no specific difficulties that met our criterion of being made by an average of at least $10 \%$ of either population of students.

One overall important observation coming from Table I is that for each skill only a small fraction of students

TABLE I. The mean percentage of responses indicating given difficulty, the percent of students indicating given difficulty on at least $75 \%$ of the relevant items, and the percent of students who never indicated given difficulty. Some response percentages from the algebrabased students were not included because of a confounding question format in the assessment used.

\begin{tabular}{|c|c|c|c|c|c|c|c|}
\hline \multirow[b]{2}{*}{ Skill } & \multirow[b]{2}{*}{ Difficulty } & \multicolumn{3}{|c|}{ Algebra-based students $(N=590)$} & \multicolumn{3}{|c|}{ Calculus-based students $(N=57)$} \\
\hline & & $\begin{array}{l}\text { Mean \% of } \\
\text { responses }\end{array}$ & $\begin{array}{l}\% \text { Students } \\
\text { indicating } \\
\geq 75 \% \text { of time }\end{array}$ & $\begin{array}{c}\text { \% Students } \\
\text { never } \\
\text { indicating }\end{array}$ & $\begin{array}{l}\text { Mean \% of } \\
\text { responses }\end{array}$ & $\begin{array}{l}\% \text { Students } \\
\text { indicating } \\
\geq 75 \% \text { of time }\end{array}$ & $\begin{array}{c}\% \text { Students } \\
\text { never } \\
\text { indicating }\end{array}$ \\
\hline Graph $T$ & $T$ is half of cycle & 17.9 & 4.9 & 70.2 & 3.1 & 0.0 & 93.0 \\
\hline Graph $f$ & $f$ equal to $T$ & 26.3 & 12.0 & 39.5 & 19.7 & 10.5 & 57.9 \\
\hline Graph $\omega$ & $\omega$ equal to $T$ & 21.9 & 5.9 & 42.4 & 21.5 & 8.8 & 52.6 \\
\hline Graph $\omega$ & $\omega$ equal to $T / 2$ & 19.1 & 3.2 & 58.5 & 9.4 & 1.8 & 80.7 \\
\hline$\omega=2 \pi / T$ & Answer is given parameter & $\ldots$ & $\ldots$ & & 10.1 & 5.3 & 77.2 \\
\hline$\omega=2 \pi / T$ & $\begin{array}{l}\text { Answer is inverse of given } \\
\text { parameter }\end{array}$ & $\cdots$ & $\cdots$ & . & 18.4 & 15.8 & 71.9 \\
\hline$\omega=2 \pi f$ & Answer is given parameter & $\cdots$ & $\cdots$ & $\cdots$ & 25.4 & 21.1 & 61.4 \\
\hline Eq. $\omega$ & Treat $\omega$ as $2 \pi / \omega$ & 19.4 & 5.4 & 47.3 & 13.6 & 5.3 & 64.9 \\
\hline Eq. $\omega$ & Treat $\omega$ as $1 / \omega$ & 20.7 & 6.8 & 47.6 & 14.0 & 1.8 & 57.9 \\
\hline Eq. $T$ & Treat $\omega$ as $T$ & 34.1 & 23.7 & 35.9 & 15.8 & 7.0 & 66.7 \\
\hline Eq. $T$ & Treat $\omega$ as $1 / T$ & 20.0 & 8.3 & 50.5 & 17.1 & 8.8 & 64.9 \\
\hline Eq. $f$ & Treat $\omega$ as $f$ & 38.5 & 26.9 & 28.5 & 31.1 & 22.8 & 47.4 \\
\hline Eq. $f$ & Treat $\omega$ as $1 / f$ & 20.4 & 8.0 & 49.8 & 13.2 & 8.8 & 71.9 \\
\hline
\end{tabular}


consistently indicated a specific difficulty compared to students who indicated the difficulty just one or two times (out of four). For example, only about $12 \%$ of algebrabased students consistently indicated that the frequency is the period in the graph $f$ skill, while over $60 \%$ answered this way once or twice. Thus it appears that these difficulties may be more often a lack of knowledge, careless error, or simple oversight than a deeply held belief. Our pilot interviews and observations from the group work training of Sec. IV support this idea.

\section{Finding the period from a graphical representation}

When determining the period from a graphical representation, some students thought that the period was half of the actual period, for example, the time from peak to trough. This difficulty was more prevalent among the algebra-based physics students than among the calculusbased physics students. In the algebra-based course, roughly $30 \%$ of the students thought the half period was the actual period on at least one of the four questions while roughly $18 \%$ responded this way for each question.

\section{Finding the frequency from a graphical representation}

When determining the frequency from a graphical representation, the most common incorrect response was to confuse the frequency with the period. This error was frequent, with about $60 \%$ of algebra-based students and $40 \%$ of calculus-based students making this error at least once (out of 4 items).

\section{Finding the angular frequency from a graphical representation}

Students experienced two types of difficulties when trying to find the angular frequency from a graphical representation. The most common difficulty was confusing the angular frequency with the numerical value of the period. This difficulty was approximately equally common in both the algebra- and calculus-based students. While less than $10 \%$ of the students in either the algebra- or calculusbased course made the error repeatedly, nearly half of the students thought the angular frequency was equal to the period on at least one of the questions.

The second and less common difficulty students experienced was indicating that the angular frequency was equal to half of the numerical value of the period.

\section{Finding the angular frequency or period using $\omega=2 \pi / T$}

As mentioned earlier, when using the equation $\omega=2 \pi / T$, students scored equally well regardless of whether the question provided the angular frequency and asked for the period or provided the period and asked for the angular frequency. In addition, the incidences of the difficulties do not appear to be dependent on which parameter is provided.

Students encountered two main difficulties when trying to apply the relationship $\omega=2 \pi / T$. First, the students assumed that the parameter in question would be equal to the parameter given. For example, if the student were provided with $\omega=\pi / 2 \mathrm{rad} / \mathrm{s}$, the most common difficulty was to respond that $T=\pi / 2 \mathrm{~s}$. This could be seen as the confusion of angular frequency and period discussed in previous difficulty \#3. Since the algebra-based students were administered these questions in multiple-choice format with two parameters given in each answer, the algebrabased students were excluded from this skill.

Second, some student responses indicated that the period and angular frequency were related by $\omega=1 / T$, which resembles the relationship between the period and frequency. One possible explanation is that some students may be confusing frequency and angular frequency. Some support for this explanation comes from the next discussed difficulty.

\section{Finding the angular frequency or frequency using $\omega=2 \pi f$}

As with the previous skill, the students scored equally well on questions utilizing this skill regardless of whether the frequency or angular frequency was provided and encountered the main difficulty at a similar rate regardless of which parameter was provided in the question. The main difficulty using the formula $\omega=2 \pi f$ was for the student to respond that the frequency and angular frequency had the same value. About $20 \%$ of calculus-based students consistently answered this way.

\section{Finding the angular frequency from a mathematical representation}

On items asking the student to identify the angular frequency from an equation of the form $x(t)=\cos (\omega t)$, students encountered two difficulties related to identifying what each number in the cosine equation represented. First, most students in both algebra- and calculus-based classes appeared to be aware that the coefficient of the time variable $t$ was the value of interest, but they often incorrectly identified this coefficient as the period (rather than the angular frequency). For example, if the students were given the equation $x(t)=3 \cos (2 \pi t / 5+\pi / 4)$, many would use $T=2 \pi / 5 \mathrm{~s}$ with the standard relation $\omega=$ $2 \pi / T$ to find the angular frequency.

Second, some students identified the coefficient as the inverse of the angular frequency. For example, if the student were given the equation $x(t)=3 \cos (2 \pi t / 5+\pi / 4)$, the student would report the angular frequency as $\omega=5 /(2 \pi) \mathrm{rad} / \mathrm{s}$. Both of these errors occurred in roughly equal proportions (around 15\%-20\%) for both populations. 


\section{Finding the period or frequency from a mathematical representation}

Since students encountered the same type of difficulties when determining the period or the frequency from an equation of the form $x(t)=\cos (\omega t)$, we discuss both of them in this section. For both skills, the most common difficulty was to identify the coefficient of the time variable $t$ with the parameter in question. For example, if the student were presented with the equation $x(t)=$ $3 \cos (2 \pi t / 5+\pi / 4)$, the student would report the period as $2 \pi / 5 \mathrm{~s}$ when asked for the period and the frequency as $2 \pi / 5 \mathrm{~Hz}$ when asked for the frequency; this was observed on both free response and multiple choice questions and observed in interviews. Additionally, some students identified the coefficient as the inverse of the parameter in question. Returning to the previous example, the student would have reported the period as $5 /(2 \pi) \mathrm{s}$ and the frequency as $5 /(2 \pi) \mathrm{Hz}$. These difficulties were the most frequently observed and many students (often about onethird) consistently responded in one of these incorrect ways, and this indicates that this difficulty may be one of the more difficult ones to overcome.

\section{ANALYSIS OF HIERARCHY}

Given that we have identified nine essential skills for working with period, frequency, and angular frequency, and that we have found that many, if not most, students are not proficient with these skills, a natural and pedagogically practical question arises as to whether these skills fit within a hierarchical structure. In short, is proficiency in a particular skill or set of skills necessary for proficiency in another given skill?

We have two reasons to believe that there is at least an empirical hierarchy among the skills. The first reason comes from the results of the previous section: there is variability in average performance of skills, thus one might suspect that the skills with low average performance may require mastery of the skills with high average performance. The second reason is more theoretical. Given that some parameters cannot be directly "read off" from a representation but rather involve two steps, we expect to find hierarchical relations among the skills. For example, the angular frequency cannot be determined directly from a graphical representation. Instead, the period would need to be determined from the graphical representation and then the relation $\omega=2 \pi / T$ would need to be applied. Therefore, if a student understands how to determine the angular frequency from a graphical representation, then the student would also have needed to understand how to determine the period from a graphical representation and how the period and angular frequency are related. In the context of our essential skills framework, determining hierarchies could allow for improved training modules since students could practice the prerequisite skills first.
In this section we will first review a more intuitive process for determining hierarchies and discuss the results from this process. Then we will apply a more formalized process and a corresponding computer algorithm to the same set of data to help confirm our results.

\section{A. Simple multiple cross tabulation analysis}

The typical method used to formally determine the existence of hierarchies is to begin with an examination of cross tabulations of (binary) mastery achievement for all pairs of skills [16-19]. For example, if two skills, $A$ and $B$, are related hierarchically with skill $A$ implying skill $B$ $(A \rightarrow B)$, then there should be no students who have mastered $A$ but have not mastered $B$. Therefore, the notation $A \rightarrow B$ can be thought of as "mastering $A$ implies mastering $B$ " and its logical equivalent, "not mastering $B$ implies not mastering $A$." In this example, the contingency table shown in Table II of the mastery of the two skills would have cell $c$ equal to zero, that is, there are zero counterexamples that mastery of $A$ implies mastery of $B$. In real data settings, however, student responses are commonly inconsistent, and inevitably, there are counterexamples. Therefore, in order to show evidence supporting a hierarchy $A \rightarrow B$ we adopt the more qualitative and intuitive idea that the table should be consistent with the constraints $a \gg c, d \gg c$, and $b \gg c$ (cf. Refs. [18,20]). A more formal process for dealing with counterexamples is pursued in Sec. III. D. For this analysis, we operationally define that a student has "mastered" a skill if the student answered $75 \%$ of the items about that skill correctly.

An example from our data from the algebra-based physics course is shown in Tables III-V. Here, the relatively low counts in the lower-left off-diagonal cell in each data

TABLE II. Sample cross tabulation for the mastery of two hypothetical skills. If $a \gg c, d \gg c$, and $b \gg c$, then skill $A \rightarrow$ skill $B$.

\begin{tabular}{lcc}
\hline \hline & \multicolumn{2}{c}{ Skill $B$} \\
\cline { 2 - 3 } Skill $A$ & Not mastered & Mastered \\
\hline Not mastered & $a$ & $b$ \\
Mastered & $c$ & $d$ \\
\hline \hline
\end{tabular}

TABLE III. Cross tabulation for the mastery of graph $\omega$ and calculate $\omega$ or $T$ skills. Here we see indication of the hierarchy graph $\omega \rightarrow$ calculate $\omega$ or $T$.

\begin{tabular}{lcc}
\hline \hline & \multicolumn{2}{c}{ Calculate $\omega$ or $T$} \\
\cline { 2 - 3 } Graph $\omega$ & Not mastered & Mastered \\
\hline Not mastered & 286 & 162 \\
Mastered & 19 & 123 \\
\hline \hline
\end{tabular}


TABLE IV. Cross tabulation for the mastery of graph $T$ and graph $\omega$ skills. Here we see indication of the hierarchy graph $\omega \rightarrow \operatorname{graph} T$.

\begin{tabular}{lcc}
\hline \hline & \multicolumn{2}{c}{ Graph $T$} \\
\cline { 2 - 3 } Graph $\omega$ & Not mastered & Mastered \\
\hline Not mastered & 152 & 296 \\
Mastered & 24 & 118 \\
\hline \hline
\end{tabular}

TABLE V. Cross tabulation for the mastery of graph $T$ and calculate $\omega$ or $T$ skills. Here we see indication of the hierarchy calculate $\omega$ or $T \rightarrow$ graph $T$.

\begin{tabular}{lcc}
\hline \hline & \multicolumn{2}{c}{ Graph $T$} \\
\cline { 2 - 3 } Calculate $\omega$ or $T$ & Not mastered & Mastered \\
\hline Not mastered & 117 & 188 \\
Mastered & 59 & 226 \\
\hline \hline
\end{tabular}

table helps to lend support to the hierarchies graph $\omega \rightarrow$ calculate $\omega$ or $T$ and graph $\omega \rightarrow$ graph $T$. That is, for a student to have mastered finding the angular frequency from a graphical representation, the student must have also mastered applying the relation between the period and angular frequency: graph $\omega \rightarrow$ calculate $\omega$ or $T$. Table III supports this relation because only 3\% (19/590) of the cases run counter to this hierarchy, and the odds of mastery of graph $\omega$ is 11.4 times greater if calculate $\omega$ or $T$ is mastered $(95 \%$ confidence interval $[6.8,19.2])$. Similarly, the student must have also mastered finding the period from a graphical representation graph $\omega \rightarrow$ graph $T$. Table IV shows that only $4 \%(24 / 590)$ of the students respond counter to this hierarchy, and the odds of mastery of graph $\omega$ is 2.5 times greater if graph $T$ is mastered $(95 \%$ confidence interval [1.6, 4.1]).

Finally, the data in Table V suggest that a student must have mastered finding the period from a graphical representation before the student can master applying the relation between the period and the angular frequency, namely, calculate $\omega$ or $T \rightarrow$ graph $T$, but the relationship does not appear to be quite as strong: about $11 \%(59 / 540)$ of the students respond counter to this hierarchy and the odds of mastery of calculate $\omega$ or $T$ is 2.4 times greater if graph $T$ is mastered (95\% confidence interval $[1.7,3.4])$. Nonetheless, the data do indicate some support for the overall hierarchy graph $\omega \rightarrow$ calculate $\omega$ or $T \rightarrow$ graph $T$. Keep in mind, however, that overall we are not limiting ourselves to a strictly linear hierarchy. There may be branches and more complex structure.

\section{B. Results from multiple cross tabulation analysis}

We used two somewhat informal methods to build an intuitive picture of the hierarchical structure of the nine

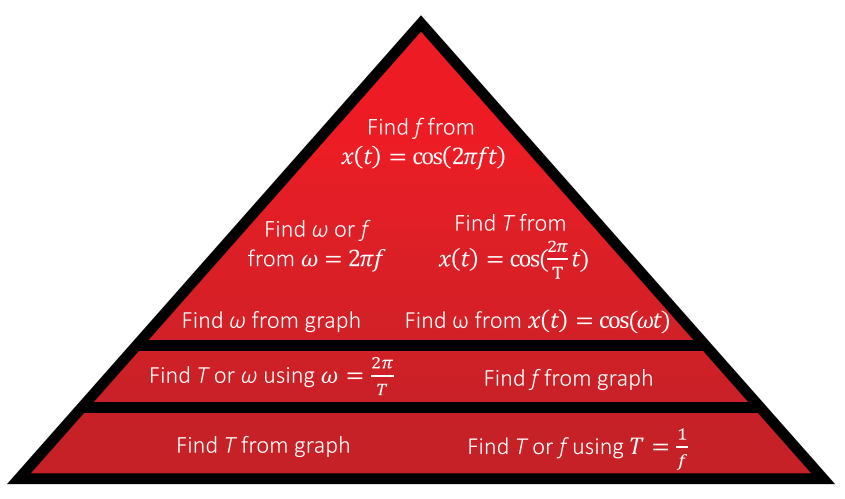

FIG. 3. A simple, informal hierarchy constructed using cross tabulations. Skills on lower levels are prerequisite to the skills on higher levels. Skills on the same level are not necessarily equivalent. Only algebra-based physics students are included in the figure.

skills. The first method was to expand upon the method in Sec. III. A and examine the cross tabulations of all possible pairs of skills to determine whether there were any hierarchies between any two skills. The results from looking across all possible contingency table pairs for the algebra-based students are shown in Fig. 3. We found that the nine skills can be divided into three levels, where skills at the same level did not show any hierarchical relations among themselves. The first level consists of the definitional skills finding the period from a graphical representation and relating the period and frequency using $T=1 / f$. The second level consists of determining the frequency from a graphical representation and relating the period and angular frequency using the equation $\omega=2 \pi / T$. The top level consists of all other skills involving the angular frequency. In addition, there were a few students (5\%), who did not master any skills.

To help support this informal analysis, we used a second informal and intuitive method, which involved the percentage of students mastering each skill, as shown in Table VI. The hierarchy in Fig. 3 is consistent with the percentage of students having mastered each skill in that

TABLE VI. Percent of the algebra-based students having mastered each of the nine skills.

\begin{tabular}{lc}
\hline \hline Skill & Percent \\
\hline Find $f$ from $x(t)=\cos (2 \pi f * t)$ & 17 \\
Find $T$ from $x(t)=\cos (2 \pi / T * t)$ & 23 \\
Find $\omega$ from graph & 24 \\
Find $\omega$ or $f$ using $\omega=2 \pi f$ & 28 \\
Find $\omega$ using $x(t)=\cos (\omega t)$ & 33 \\
Find $f$ from graph & 38 \\
Find $T$ or $\omega$ using $\omega=2 \pi / T$ & 48 \\
Find $T$ from graph & 70 \\
Find $T$ or $f$ using $T=1 / f$ & 72 \\
\hline \hline
\end{tabular}


the skills mastered by more students are on a lower level in the hierarchy than the skills mastered by fewer students.

Preliminary results for the calculus-based students suggest a hierarchy consistent with that in Fig. 3. However, due to the small sample size, we do not formally present that analysis here.

\section{Limitations of the informal cross tabulation analysis}

While the cross tabulation method is effective for creating a general description and intuition of the hierarchy, it suffers from a few inherent problems. First, the criteria for determining a hierarchy (e.g. the count in one off-diagonal cell is "much less" than the others) is not well defined. Put another way, there is no formal way to account for cases that run counter to a proposed hierarchy. These counterexamples may occur for a variety of reasons including guessing, inadvertent errors, variability due to contextual factors, or possibly student confusion or confounding of variables, as evidenced by our observation that sometimes a student would answer two identical problems (except for the values) in two distinct manners. For example, for graph $\omega$ problems, the student would answer one of the problems correctly but answer another as if the question had (ostensibly) asked for the period. Second, there is no formal measure to determine how well the data fits any proposed hierarchy, or how to decide which potential hierarchy fits the best. Finally, there is no formal way to account for all possible branchings in the hierarchy. To account for these limitations, we employ the method of item tree analysis, discussed in Sec. III. D.

\section{Item tree analysis (ITA)}

Item tree analysis was first developed in 1974 by Van Leeuwe [17] and since then, multiple modified, inductive versions have been created $[19,21,22]$. Each of the various versions of ITA are based on the ideas of knowledge space theory which was introduced by Doignon and Falmagne in 1985 [23]. In brief, knowledge space theory provides a framework for knowledge and skills that describes a knowledge (or skill) domain in terms of $N$ problems that span the domain, and the subset of problems a given person can successfully solve defines a knowledge state. The set of all (empirically) possible knowledge states is the knowledge structure. It is this framework of knowledge structure that can be used to formally describe a hierarchy among the $N$ problems of the domain.

All the Inductive ITA methods employ the framework of knowledge space theory and follow a similar procedure, which will be outlined here. For a full explanation of the algorithm, see Ref. [22]. First, suppose an assessment has $m$ items and is completed by $n$ subjects. Form the $n$ by $m$ matrix $D$ that contains the responses to the $m$ items in a dichotomous manner. Now consider pairs of items, $i$ and $j$, and find all pairs such that $j \rightarrow i$ and the number of counterexamples, $b_{i j}$, is zero. From these pairs, construct a hierarchy, which will be called $H_{0}$. Next, find all pairs of items such that $j \rightarrow i$ and the number of counterexamples, $b_{i j}$, is no more than 1 . From these pairs, construct a hierarchy, which will be called $H_{1}$. This process is repeated with the number of allowed counterexamples increasing by 1 until the maximum number of possible counterexamples is reached, that is the number of counterexamples is equal to the number of subjects, $n$.

Once all the hierarchies have been generated, the expected number of counterexamples, $b_{i j}^{*}$ for each relation $j \rightarrow i$ is computed. How this expected number of counterexamples is computed varies based on which version of inductive ITA is utilized. Next, the sum $C \Sigma_{i \neq j}\left(b_{i j}-b^{*}{ }_{i j}\right)^{2}$, where $C$ is some constant, is computed for each hierarchy $H_{k}$, which represents the discrepancy between the observed and expected number of counterexamples for all relations and is called $\operatorname{diff}\left(H_{k}, D\right)$, where $k=0,1,2, \ldots, n$. As Sargin and Ünlü [22] note, the constant is picked to be $C=1 /[n(n-1)]$, but any other constant scale factor would not change the results. Finally, the hierarchy that best fits the data is chosen, which is the hierarchy that minimizes the value of $\operatorname{diff}\left(H_{k}, D\right)$. In order to implement the inductive ITA algorithm, the DAKS package for $\mathrm{R}$ was used [24].

\section{E. Item tree analysis results}

Sargin and Ünlü $[22,25]$ find that the corrected algorithm and the minimized corrected algorithm perform equally well for large sample sizes and outperform the original inductive ITA algorithm, in that these two algorithms detect less erroneous relations than the original inductive ITA algorithm does. Therefore, we utilized the former algorithms rather than the latter algorithm. Since item tree analysis has limited validity for small sample sizes, we only present results from the algebra-based students. The corrected and minimized corrected algorithms find identical hierarchical relations so we only report the results from the minimized corrected algorithm here.

The resulting hierarchy for the algebra-based students is shown in Fig. 4. The error rate for the resulting hierarchy is 0.20 , which can be thought of as roughly the average ratio of the number of counterexamples to the hierarchical relations to the number of examples consistent with the hierarchical relations. The judgment as to whether this is a large or small error rate is somewhat subjective, though it does seem that 0.20 (which is in effect a 1:5 odds ratio) does indicate a fair amount of counterexamples observed in this domain. In contrast, it also indicates that, overall, the hierarchy is accurate roughly $84 \%(5 / 6)$ of the time. While the hierarchy shown in Fig. 4 is more complex than the more informally derived hierarchy shown in Fig. 3, the general structure is similar to that found by using the cross tabulation approach. 


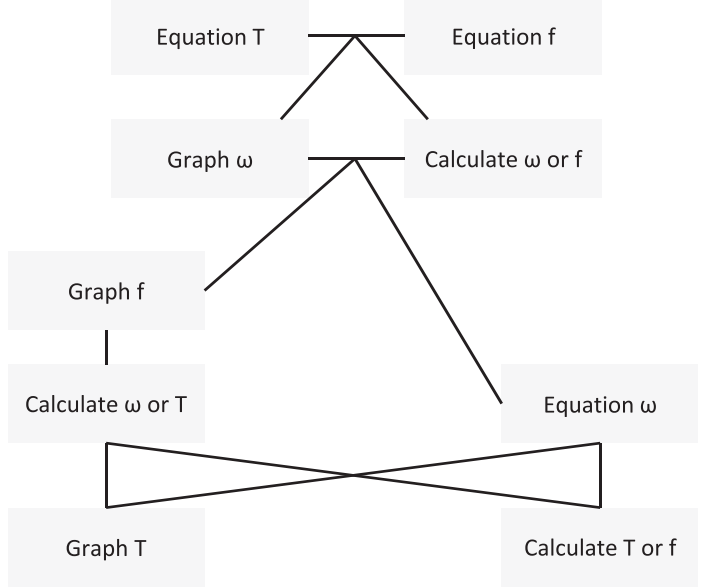

FIG. 4. Hierarchy of period, frequency, and angular frequency skills for the algebra-based students obtained using item tree analysis and the DAKS package for R.

\section{DEVELOPMENT OF AN INTERVENTION}

In this final study, we used the results of the first parts of this study to develop a group work "tutorial" activity to improve students' ability to determine the period, frequency, and angular frequency from mathematical and graphical representations. In addition, we hope to use this material to inform the construction of online essential skills modules in future work.

\section{A. Methods}

This study uses students from the same calculus-based physics course pool described in Sec. II A. Students were randomly assigned to either the tutorial or the nontutorial (control) condition when they entered our lab. Upon the start of the in-person sessions, the students in the tutorial condition were randomly placed in groups of three, with up to six students participating per $1 \mathrm{~h}$ session. In the case that a full group of three students could not be formed, students were randomly selected to form a group of three and the remaining students were assigned to the control condition in which participants did not receive the tutorial, but instead completed physics tasks unrelated to period, frequency, and angular frequency. These nontutorial group students were sent to a separate room so that they would not hear any discussion occurring during the tutorial. Upon completing the tasks unrelated to period, frequency, and angular frequency, the nontutorial students completed a period, frequency, and angular frequency assessment, which will be described in the following.

The tutorial group students began by answering questions (on paper) asking them to consider the definition and units of period, frequency, and angular frequency. The students were then asked to use those definitions to write the formulas relating all three quantities $(T=1 / f$, $\omega=2 \pi / T, \omega=2 \pi f$ ) and then to use those formulas to check that the formulas produced the correct values when given one of the parameters. Next, the students were asked to determine the frequency of a mass on a spring from a graphical representation and to mark half and a quarter of a cycle. Finally, the students were asked to find the period, frequency, and angular frequency from a graphical representation (with the parameters picked so that the three quantities could easily be identified) and to use their three equations to show that all three answers were consistent with each other. The students answered two such questions. The tutorial was designed to be completed within $30 \mathrm{~min}$ or roughly half a recitation session, though this experiment was not actually conducted in a recitation session, and was completed with little to no intervention by the room proctor. The proctor only intervened when the students did not understand what a question was asking. Our general observation was that almost all students were engaged in the activity and discussed the questions in the group. The students were not told the correct answers to the tutorial questions once they had completed the tutorial.

Upon the completion of the tutorial, the students were individually administered an assessment that assessed six of the nine skills (graph $T$, graph $f$, graph $\omega$, calculate $T$ or $f$, calculate $\omega$ or $T$, and calculate $\omega$ or $f$ ) with 28 items. The calculation questions were free response while the questions involving graphical representations were presented as free response or multiple choice. The multiple-choice questions either presented the student with a graphical representation and asked them to determine the period, frequency, or angular frequency or presented the student with one of the three parameters and asked the student to select the graphical representation that correctly depicted the parameter. In addition to these 28 relevant items, the assessment contained four proportional reasoning items involving the maximum speed, maximum acceleration, period, and frequency of a harmonic oscillator, which were not trained on the tutorial. The tutorial and nontutorial students completed the same assessment. Students who did not answer at least half of the questions on the assessment were excluded from the analysis, as we do not believe these students put an honest effort into answering the questions. This left 55 students in the tutorial group and 65 students in the nontutorial group. In order to confirm the equivalency of the two randomly assigned groups, a comparison of the final course grades of the two groups was computed and revealed that the average ( $z$ score) of the students who participated in the tutorial was not statistically different from the $z$ score of the students in the nontutorial control group $[t(118)=0.162, p>0.1]$.

\section{B. Results}

The tutorial group, with a mean score of $81 \%$, scored significantly better than the nontutorial group, with a mean score of $57 \%[t(98)=5.258, p<0.001$, effect size $d=1.04]$. The distribution of the scores on the 28 relevant 


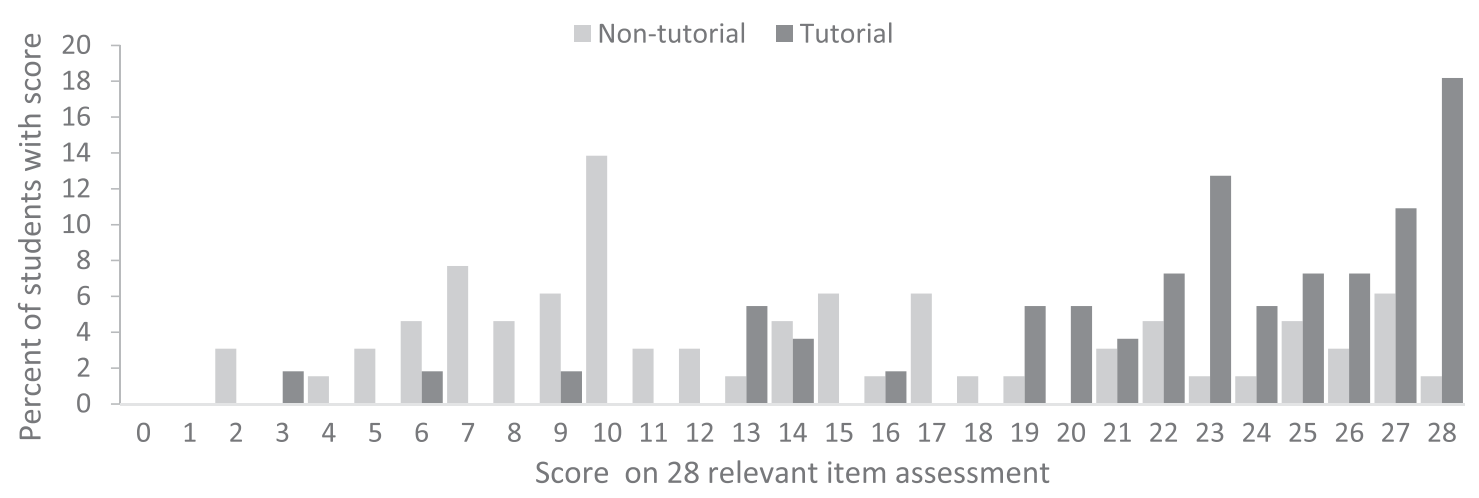

FIG. 5. Distribution of scores for the tutorial and nontutorial groups on the 28 items relevant to the tutorial. The percentages are based on the students within each treatment.

items for both the tutorial group and the nontutorial group are shown in Fig. 5, and show the dramatic difference in scores.

To determine whether any specific skills were driving the improvement, we then examined the tutorial and nontutorial students' scores on each of the six skills; these are shown in Fig. 6. Students in the tutorial condition performed better than the control students did across all six skills tested (non-Bonferonni corrected $p=0.003$ for graph $T$ and $p<0.001$ for the other five skills). Thus, it appears that all six skills that were trained contributed to the increased performance by the tutorial group.

In contrast, we compared the tutorial and nontutorial students' scores on the four items not covered by the tutorial, which are also shown in Fig. 6. A $t$ test shows that there is no statistical difference between the two groups' scores $[t(118)=0.903 p>0.1]$, suggesting that the differences in scores between the tutorial and nontutorial groups can be attributed to the tutorial and not to a difference in the groups themselves.

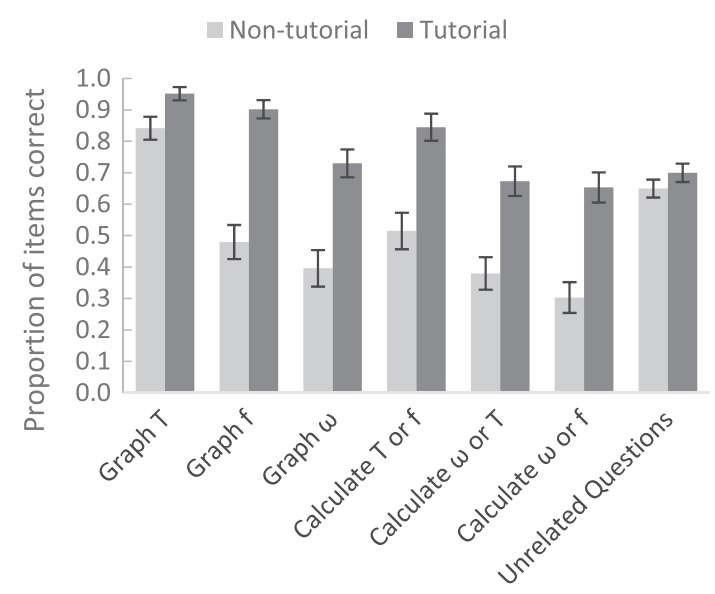

FIG. 6. A comparison between the proportion correct for tutorial students vs nontutorial students for each $T, f, \omega$ skill, and for four unrelated physics questions.

\section{CONCLUSIONS AND DISCUSSION}

The overall context of this study is that, through a series of pilot tests and interviews, we categorized nine essential skills needed for determining the period, frequency, and angular frequency from commonly used graphical and mathematical representations for a generic harmonic oscillator scenario. These skills are simple, fundamental, and necessary for several areas of the physics curriculum.

We present three main conclusions from this study. The first conclusion is that, even postinstruction, only about $20 \%-40 \%$ of students have mastered most of the nine skills for determining period, frequency, and angular frequency. There are a couple of skills in which most students are fairly proficient, namely, determining the period of a harmonic oscillator from a position vs time graph, or calculating period given frequency or vice versa, but even for the latter skill, students demonstrated only about $75 \%$ overall accuracy. For such simple and essential skills, it would seem natural for instructors to expect (or assume) proficiency to be much closer to $100 \%$. However, the nature of student errors are likely familiar to instructors. For example, students often make errors of factors of 2, inversion errors, and confusion of variables. It is also worth noting that while we have reported here some common errors, we also found that some students demonstrated significant diversity in their answers, perhaps due to uncertainty in whether their path to the solution was a correct one. Specifically, the free response questions generated a wide distribution of answers that did not seem to have a specific pattern. For example, among the calculus-based students, there were at least 15-20 unique responses for each question asking students to determine the frequency or angular frequency from a graphical representation and between 10 and 20 unique responses for each question asking students to determine the period, frequency, or angular frequency from a mathematical representation. Given the small sample sizes of the calculus-based students in the study (about 60 per study), this represents a sizable population of students who appear to be 
using unspecified (or unspecifiable?) methods for obtaining an answer rather than using the normatively relevant knowledge and skills. Thus, it appears that there are three groups of students: those who have mastered a skill, those who have not mastered a skill but whose answers are informed by knowledge of the period, frequency, and angular frequency, and those whose answers are not informed by any ostensibly relevant knowledge.

Further, while the average percent of students encountering a difficulty is informative of how often a difficulty occurs, the percent of students never encountering the difficulty is also informative. On most of the skills, roughly half of the algebra-based students experienced each difficulty at least once while roughly one-third of the calculusbased students experience each difficulty at least once. Therefore, it seems that even if students do answer some of the questions regarding a particular skill correctly, many still may have some uncertainty on whether what they are doing is correct, or they may not be using methods that avoid unmindful errors.

The second conclusion is that, based on student answering patterns, one can meaningfully and quantifiably arrange the nine skills in a hierarchical structure, with some skills being necessary prerequisites for other skills. We determined a hierarchy with an intuitive, semi-quantitative method, and also with the formal item tree analysis process and found them to be consistent, raising our understanding and confidence in the validity of the hierarchy. Specifically we found that all nine skills are linked in a (roughly) four-level, nonlinear tree. We found that the skills of determining the period from a graph and calculating period from frequency are prerequisite skills for all other skills, and determining period or frequency from an equation of the form $x(t)=\cos (\omega t)$ are the highest level skills. The method of item tree analysis is rarely if ever used in education research, but appears to be a very useful tool.

The third conclusion of this study is that students demonstrate dramatic gains (1 standard deviation) in accuracy in these skills with only $30 \mathrm{~min}$ of targeted practice in a group setting. One limitation of this study is that we do not know how long this gain in skills was retained. Nonetheless, this relatively easy gain further characterizes these skills and makes them excellent candidates for online, spaced, mastery practice with feedback (cf. Refs. [14,15]). Such practice could increase the fluency and retention of these skills and help students to solve more complex problems. It is interesting to consider how the observed hierarchy of the skills might inform such targeted practice and is an interesting topic for future study. Certainly, this study suggests that since determining the period from a graph and proficiency with the periodfrequency relationship are on the bottom of the hierarchy, practice with these skills should be the focus of early practice.

\section{ACKNOWLEDGMENTS}

Funding for this research was provided by the Center for Emergent Materials: an NSF MRSEC under Grant No. DMR-1420451.
[1] D. C. Giancoli, Physics: Principles with Applications, 7th ed. (Pearson Education, Upper Saddle River, NJ, 2014).

[2] J. Walker, D. Halliday, and R. Resnick, Fundamentals of Physics, 10th ed. (Wiley, Hoboken, NJ 2014).

[3] H. D. Young and R. A. Freedman, University Physics with Modern Physics (Pearson Education, Upper Saddle River, NJ, 2014).

[4] L. C. McDermott, M. L. Rosenquist, and E. H. Van Zee, Student difficulties in connecting graphs and physics: Examples from kinematics. Am. J. Phys. 55, 503 (1987).

[5] R. J. Beichner, Testing student interpretation of kinematics graphs, Am. J. Phys. 62, 750 (1994).

[6] T. Wan, P. J. Emigh, G. Passante, and P. S. Shaffer, Student understanding of period in introductory and quantum physics courses, Proceedings of the Physics Education Research Conference 2016, Sacramento, CA, (AIP, New York, 2016), pp. 380-383, DOI: 10.1119/ perc.2016.pr.090.

[7] W. A. Turner and G. W. Ellis, and R. J. Beichner, A Comparison of Student Misconceptions in Rotational and Rectilinear Motion, Proceedings of the 2014 ASEE
Annual Conference \& Exposition, Indianapolis (ASEE, Washington DC, 2014), https://peer.asee.org/19926.

[8] B. S. Ambrose, Probing student reasoning and intuitions in intermediate mechanics: an example with linear oscillations, AIP Conf. Proc. 883, 30 (2007).

[9] N. Tongnopparat, J. Poonyawatpornkul, and P. Wattanakasiwich, Investigation of student reasoning about harmonic motions, Proceedings of the 12th Asia Pacific Physics Conference (Physical Society of Japan, Tokyo, 2014), p. 017033, DOI: 10.7566/JPSCP.1.017033.

[10] M. Kryjevskaia, M. R. Stetzer, and P. R. Heron, Student understanding of wave behavior at a boundary: The relationships among wavelength, propagation speed, and frequency, Am. J. Phys. 80, 339 (2012).

[11] M. Kryjevskaia, M. R. Stetzer, and P. R. Heron, Student difficulties measuring distances in terms of wavelength: Lack of basic skills or failure to transfer?, Phys. Rev. ST Phys. Educ. Res. 9, 010106 (2013).

[12] M. Wittmann, R. N. Steinberg, and E. F. Redish, Understanding and affecting student reasoning about sound waves, Int. J. Sci. Educ. 25, 991 (2003). 
[13] L. G. Rimoldini and C. Singh, Student understanding of rotational and rolling motion concepts, Phys. Rev. ST Phys. Educ. Res. 1, 010102 (2005).

[14] B. D. Mikula and A. F. Heckler, Framework and implementation for improving physics essential skills via computer-based practice: Vector math, Phys. Rev. Phys. Educ. Res. 13, 010122 (2017).

[15] N. Schroeder, G. Gladding, B. Gutmann, and T. Stelzer, Narrated animated solution videos in a mastery setting, Phys. Rev. ST Phys. Educ. Res. 11, 010103 (2015).

[16] W. M. Bart and D. J. Krus, An ordering-theoretic method to determine hierarchies among items, Educ. Psychol. Meas. 33, 291 (1973).

[17] J. F. Van Leeuwe, Item tree analysis, Nederlands Tijdschrift voor de Psychologie en haar Grensgebieden 29, 475 (1974).

[18] R. Rosenblatt and A. F. Heckler, Systematic study of student understanding of the relationships between the directions of force, velocity, and acceleration in one dimension, Phys. Rev. ST Phys. Educ. Res. 7, 020112 (2011).
[19] M. Schrepp, A method for the analysis of hierarchical dependencies between items of a questionnaire, Methods Psychol. Res. Online 19, 43 (2003).

[20] R. T. White and R. M. Clark, A test of inclusion which allows for errors of measurement, Psychometrika 38, 77 (1973).

[21] M. Schrepp, On the empirical construction of implications between bi-valued test items, Mathematical Social Sciences 38, 361 (1999).

[22] A. Sargin and A. Ünlü, Inductive item tree analysis: Corrections, improvements, and comparisons, Mathematical Social Sciences 58, 376 (2009).

[23] J. P. Doignon and J. C. Falmagne, Spaces for the Assessment of Knowledge, International Journal of Man-Machine Studies 23, 175 (1985).

[24] A. Unlü and A. Sargin, DAKS: an R package for data analysis methods in knowledge space theory. J. Stat. Softw. 37, 1 (2010)

[25] A. Ünlü and M. Schrepp, Untangling comparison bias in inductive item tree analysis based on representative random quasi-orders, Mathematical Social Sciences 76, 31 (2015). 